\title{
Impact of Thermal Annealing on the Semicrystalline Nanomorphology of Spin-Coated Thin Films of Regioregular Poly(3- alkylthiophene)s as Observed by High-Resolution Transmission Electron Microscopy and Grazing Incidence X-ray Diffraction
}

\author{
Shabi Thankaraj Salammal, Eduard Mikayelyan, Souren Grigorian, and Ullrich Pietsch
}

Solid State Physics, University of Siegen, Walter Flex Strasse-3, D-57068 Siegen, Germany

Nils Koenen and Ullrich Scherf

Macromolecular Chemistry, University of Wuppertal, D-42097, Gauss-Str. 20, Wuppertal, Germany

Navaphun Kayunkid and Martin Brinkmann*

Institut Charles Sadron, CNRS - Université de Strasbourg, 23 rue du loess, 67034 Strasbourg, France

ABSTRACT: The impact of thermal annealing on the growth of crystalline nanodomains of poly(3-alkylthiophene) (P3AT) in thin films (15-20 nm thick) was investigated as a function of length of alkyl side chain by combined low-dose highresolution transmission electron microscopy (HR-TEM) and grazing incidence X-ray diffraction (GIXD). Statistical analysis of the data yields the characteristic dimensions of the face-on oriented P3AT nanocrystals, i.e., average stem length $l_{\mathrm{c}}$ along backbones and lateral dimension perpendicular to the stems $l_{\mathrm{a}}$ along side chains. The following trends were identified: (i) in

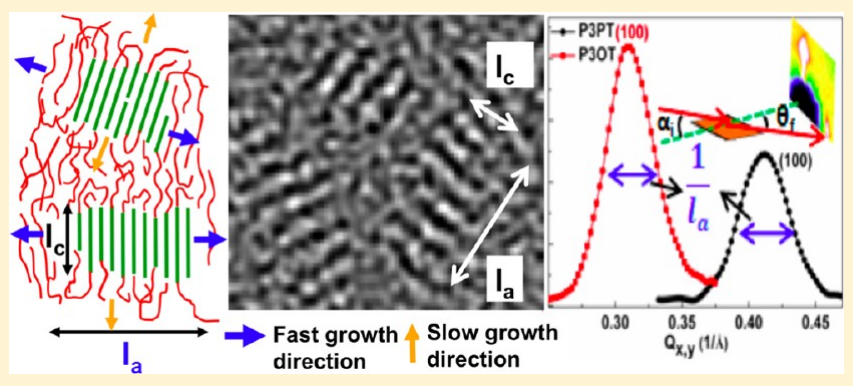
as-spin-coated films, the proportion of face-on oriented nanocrystals increases with the number of carbon atoms in the side chain, (ii) annealing favors the lateral in-plane growth of the nanocrystals along the side chain direction ( $\mathbf{a}_{\mathrm{P} 3 \mathrm{AT}}$ axis), (iii) for a given P3AT, the proportion of face-on oriented domains increases with annealing temperature, (iv) lateral growth along the $\mathbf{a}_{\mathrm{P} 3 \mathrm{AT}}$ axis is most efficient for the longer octyl side chains, and (v) thermal annealing induces only modest lamellar thickening which is limited by the poor slide diffusion of $\pi$-stacked P3AT chains as opposed to lateral growth favored by weak van der Waals interactions between layers of $n$-alkyl side chains. The increase in the population of face-on oriented crystallites, observed when the length of the side chain increases, coincides with a corresponding decrease in the field effect mobility in annealed P3AT thin films.

\section{INTRODUCTION}

Mastering the morphology and orientation of semicrystalline regioregular poly(3-alkylthiophene)s (RR-P3ATs) is an essential step to improve the performances of organic electronic devices, e.g., organic solar cells and organic field effect transistors (OFETs). ${ }^{1,2}$ From a structural point of view, the chemical incompatibility between the conjugated polythiophene backbone and alkyl side chains results in the buildup of a lamellar "self-assembled" structure in which $\pi$-stacked polythiophene backbones alternate with layers of more or less ordered alkyl side chains. ${ }^{3-5}$ P3AT films can be prepared by various methods, e.g., spin-coating, dip-coating, drop-casting, and directional epitaxial crystallization (DEC) that influences the level of crystallinity and preferred orientation of P3AT domains on the substrate. ${ }^{6-9}$ However, whatever the preparation method, the films are semicrystalline; i.e., crystalline P3AT domains coexist with amorphous interlamellar zones. In most studies, P3AT nano- crystallites adopt mainly two preferential orientations on the substrate labeled "face-on" and "edge-on", respective to the conjugated backbone lying parallel or perpendicular to the substrate plane. ${ }^{10}$ The control of these orientations is essential as they correspond to the $\pi$-stacking direction lying out-of-plane (face-on) or in-plane (edge-on) which is favorable to photovoltaic applications in the first case and for OFETs in the second case. The formation of P3AT crystallites with various orientations is mainly depending on the growth procedures and macromolecular parameters, e.g., regioregularity (RR), molecular weight $\left(M_{\mathrm{w}}\right)$, and length of the linear alkyl side chains. ${ }^{11-15}$ From the recent literature, it turns out that P3HT adopts mainly the "edge-on" orientation for thermodynamic

Received: May 4, 2012

Revised: June 18, 2012

Published: June 28, 2012 
reasons. ${ }^{6,7,13}$ "Edge-on" orientation can be favored in various manners, e.g., via substrate treatments with self-assembled monolayers (SAM) $)^{12,16,17}$ by using high boiling point solvents, e.g., 1,2,4-trichlorobenzene, ${ }^{18}$ or postgrowth annealing. ${ }^{19}$ The widely used spin-coating technique is producing kinetically trapped morphologies due to the fast evaporation of the solvent. In many instances, postgrowth annealing has been used to induce reorientation and crystallization of the films with the hope of inducing lamellar thickening as observed in the case of classical polymers, e.g., polyethylene (PE) ${ }^{20}$ Generally, thermal annealing was reported to result in the development of nanofibrillar domains as well as improved charge mobilities. ${ }^{19}$ Numerous studies have evidenced the strong impact of thermal annealing on the structure and morphology of the spin-coated films mainly by grazing incidence X-ray diffraction (GIXD) and atomic force microscopy (AFM) ${ }^{13,14}$ However, these methods do not allow a complete visualization of the semicrystalline morphology of the films, i.e., distinguish amorphous zones from crystalline lamellae. Recently, Brinkmann and co-workers have shown that transmission electron microscopy in bright field and also in low-dose high-resolution mode allows to visualize the nanomorphology of the films down to the scale of individual stacks of polythiophene chains within the crystalline domains. ${ }^{21-23}$ This is essential as charge transport is limited by the interconnectivity between crystalline domains through the amorphous interlamellar zones. $^{23}$ Accordingly, mapping the nanomorphology (interconnectivity of domains, dimensions, preferred orientation) as a function of preparation conditions is an essential step toward a better modeling and understanding of charge transport in these semicrystalline systems. Moreover, P3ATs are reported to fold, similar to the more flexible chains like $\mathrm{PE},{ }^{24-27}$ which is known to impact the growth features of semicrystalline polymers; e.g., lamellar thickening in the direction of the chains is expected upon thermal annealing. ${ }^{20}$ As yet, various studies have clearly demonstrated that P3AT chains tend to fold above a certain $M_{\mathrm{w}}$ of $10 \mathrm{kDa} .{ }^{28,29}$ Chain folds in P3AT are modeled by a sequence of 7-8 monomer units in cis conformation leading to a $2.0 \mathrm{~nm}$ wide fold. Whereas in a crystal structure like PE the interchain interactions with the next-neighbor chains are uniform in the plane perpendicular to the chain, the situation is quite different for P3ATs. P3ATs are characterized by strong $\pi-\pi$ interactions between polythiophene backbones (i.e., along the $\mathbf{b}_{\mathrm{P} 3 \mathrm{AT}}$ axis) and weak van der Waals interactions along the side chain direction $\left(\mathbf{a}_{\mathrm{P} 3 \mathrm{AT}}\right.$ axis) which makes this system significantly different from the more classical polyolefins. As yet, there has been only a limited set of studies dealing with the implications of the anisotropy of interactions on the crystallization mode of P3ATs.

The present study aims at following the semicrystalline nanomorphology of various P3AT films with $n$-alkyl side chains ranging from $n$-pentyl to $n$-octyl as a function of annealing conditions (annealing temperature $T_{\mathrm{a}}$ ). Our reseach is based in part on a statistical analysis of domain sizes both along the stem direction ( $\mathbf{c}_{\mathrm{P} 3 \mathrm{AT}}$ axis) and along $\mathbf{a}_{\mathrm{P} 3 \mathrm{AT}}$ axis using HR-TEM in lowdose mode. In particular, this study will help to identify the impact of thermal annealing on both the lamellar thickening and the lateral growth of the P3AT domains and to understand their side-chain-dependent anisotropy.

\section{EXPERIMENTAL SECTION}

The poly(3-alkylthiophene)s (P3ATs) were synthesized using the procedure documented in the literature. ${ }^{30,31}$ Prior to the polymerization, the 3-bromothiophene was alkylated using $n$-butyllithium ( $n$ $\mathrm{BuLi}$ ) and the corresponding alkyl bromide. ${ }^{30}$ The next step is dibromination with $\mathrm{N}$-bromosuccinimide (NBS) in $\mathrm{N}, \mathrm{N}$-dimethylformamide (DMF) at room temperature. ${ }^{31}$ The monomer was then polymerized via Grignard metathesis (GRIM) as reported by Loewe et al. ${ }^{32}$ The P3AT polymers such as (poly(3-pentylthiophene) (P3PT), poly(3-hexylthiophene) (P3HT), poly(3-heptylthiophene) (P3HeptT), and poly(3-octylthiophene) (P3OT) with $>99 \%$ RR were obtained. The RR and melting point of the polymers were analyzed by nuclear magnetic resonance spectroscopy (NMR) and differential scanning calorimetric (DSC) analyses, respectively. The melting temperature $\left(T_{\mathrm{m}}\right)$, polydispersity index (PDI), and annealing temperatures of the polymers are collected in Table 1 .

Table 1. Macromolecular SEC Parameters $\left(M_{n}, M_{w}\right.$, and $I=$ $\left.M_{\mathrm{w}} / M_{\mathrm{n}}\right)$ of P3AT Samples

\begin{tabular}{lcccc} 
polymers & $\begin{array}{c}\mathrm{mol} \mathrm{wt} \\
(\mathrm{kg} / \mathrm{mol})\end{array}$ & PDI & $\begin{array}{c}T_{\mathrm{m}}(\text { from DSC }) \\
\left( \pm 2^{\circ} \mathrm{C}\right)\end{array}$ & $\begin{array}{c}T_{\mathrm{m}} \text { (from X-ray data) } \\
\left( \pm 5^{\circ} \mathrm{C}\right)\end{array}$ \\
P3PT & 36.0 & 1.5 & 249 & 245 \\
P3HT & 45 & 1.2 & 229 & 235 \\
P3HeptT & 42.5 & 1.2 & 206 & 210 \\
P3OT & 39 & 1.5 & 184 & 195 \\
\hline
\end{tabular}

P3AT thin films were spin-coated on thermally oxidized $(293 \mathrm{~nm}$ thick $\left.\mathrm{SiO}_{2}\right) \mathrm{Si} / \mathrm{SiO}_{2}$ substrate. Prior to the film preparation, the substrates were cleaned in an ultrasonic bath with an aqueous solution of hellmanex (Helma), 2-propanol, and acetone prior to drying under a flow of nitrogen. P3AT solutions (2 wt \%) were prepared by dissolving the polymer in chloroform and stirring for several hours at $45{ }^{\circ} \mathrm{C}$ to achieve complete dissolution. The obtained solutions were subjected to UV-vis absorption spectroscopy to confirm the complete dissolution of polymers. $15-18 \mathrm{~nm}$ thick films were obtained after spin-coating at $2000 \mathrm{rpm}$ for $30 \mathrm{~s}$. Films were subsequently annealed for $1 \mathrm{~h}$ under a $\mathrm{N}_{2}$ atmosphere at a temperature $T_{\mathrm{a}}$ corresponding to an undercooling $\Delta T=$ $45^{\circ} \mathrm{C}$ below their corresponding melting points. The annealing duration was determined from the time dependence of the (100) X-ray peak intensity upon annealing (see below). As a matter of fact, marginal change of the (100) peak intensity was observed for annealing times above $1 \mathrm{~h}^{6}$

For TEM analysis, the P3AT thin films were covered with a thin carbon film and subsequently recovered on TEM copper grids by floating the films on a $10 \mathrm{wt} \% \mathrm{HF}$ (hydrogen fluoride) solution. TEM analysis was performed using a CM12 Philips microscope $(120 \mathrm{kV})$ equipped with a MVIII CCD camera. A modified low-dose operating mode was used as described in detail elsewhere. ${ }^{21}$ The image filtering and fast-Fourier transform (FFT) analyses were performed using the AnalySiS software. Fast Fourier filtering conditions were chosen such as to minimize ghost lattice generation as explained in ref 21 .

For the X-ray analyses we used both synchrotron and laboratory sources with different wavelengths ranging from 0.82 to $1.55 \AA$. Under GIXD conditions the incidence angle $\left(\alpha_{\mathrm{i}}\right)$ of the X-ray beam was fixed slightly above the critical angle of the polymer $\left(\alpha_{\mathrm{p}}\right)$ and below the critical angle of the substrate $\left(\alpha_{c}\right)$. Reflectivity scans were additionally performed to calculate the thickness and roughness of the films under specular conditions. Point and 2D detectors were used to probe the stacking of the crystallites by in-plane (GID) and out-of-plane (GOD) diffraction. The optimum annealing temperatures of the four polymers were determined by in situ annealing analysis using an Anton Paar DHS 900 (dome heating stage).

The mobilities of the spin-coated P3AT OFETs were measured in the saturation regime. ${ }^{33}$ Bottom contact OFETs were fabricated by spincoating the films on the patterned test beds purchased from Fraunhofer IPMS, Dresden, Germany . These end-of-line testbeds for customized organic semiconductors can be used to fabricate up to 16 OFETs on one chip $\left(15 \times 15 \mathrm{~mm}^{2}\right)$ with different channel lengths like $2.5,5,10$, and 20 $\mu \mathrm{m}$ and a width of $10 \mathrm{~mm}$. The interdigitated source and drain electrodes $(\mathrm{Au})$ were patterned on the $230 \mathrm{~nm}$ thick thermally oxidized heavily doped n-type Si substrate. The testbeds were cleaned with acetone followed by isopropanol and dried in a flow of $\mathrm{N}_{2}$. The mobility measurements were carried out under ambient conditions after drying 
the films under a $\mathrm{N}_{2}$ atmosphere for $30 \mathrm{~min}$. The mobility of the same film was remeasured after annealing at the undercooling of $45^{\circ} \mathrm{C}$ for $1 \mathrm{~h}$.

\section{RESULTS}

a. Conditions of Isothermal Annealing. In order to determine an appropriate annealing protocol for the various P3ATs films under investigation, i.e., choosing comparable undercooling conditions, the melting temperature of the films was first identified by in situ X-ray diffraction measurements using the laboratory source. To this aim, highly crystalline P3AT films drop-casted at $-30{ }^{\circ} \mathrm{C}$ and yielding an intense $\mathrm{X}$-ray scattered signal were used. Indeed, as shown in a recent study, casting of P3AT at temperatures below room temperature yields thin films with enhanced crystallinity and preferred orientation that can be improved by thermal annealing. ${ }^{6}$ For determining the melting temperature, it is essential to measure precisely the intensity of the structural peaks as a function of annealing temperature, $T_{\mathrm{m}}$. Accordingly, the P3AT films were subjected to a temperature ramp. Figure 1 shows the evolution of the (100) X-ray peak

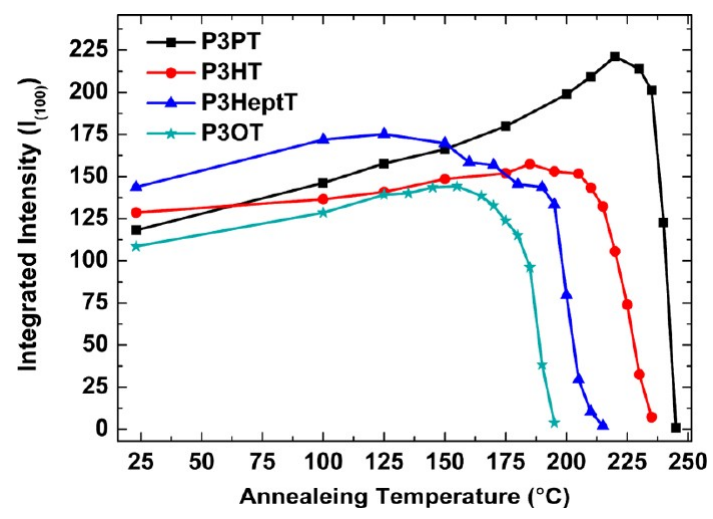

Figure 1. Dependence of the integrated intensity of the (100) X-ray peak intensity (Bragg-Brentano configuration) as a function of the annealing temperature.

intensity versus annealing temperature for various P3AT films. For each temperature step, the sample was allowed to thermalize for about $15 \mathrm{~min}$, i.e., until the intensity of the (100) peak has reached a constant value. As expected, the increase of the annealing temperature tends to induce an increase of the (100) $\mathrm{X}$-ray peak intensity before it decreases at a certain temperature approaching $T_{\mathrm{m}}$. It turns out that the longer the side chain, the lower is $T_{\mathrm{m}}$. The derived melting temperatures for the investigated P3AT samples are in good agreement with the experimental melting temperatures of P3ATs obtained by DSC (see Table 1).

Accordingly, for the comparison of the impact of thermal annealing on the morphology of various P3ATs, all samples were isothermally annealed by the same conditions, i.e., using an undercooling $\Delta T=45{ }^{\circ} \mathrm{C}$ with respect to the experimental melting temperature. The chosen $\Delta T$ reveals to be an appropriate value for the temperature-induced growth of the crystalline domains and simultaneously to avoid premelting. Regarding the duration of annealing, the kinetics of the intensity increase of the (100) reflection indicates that marginal changes occur for annealing times beyond $1 \mathrm{~h}$. Accordingly, in the following, all samples have been annealed at the specified temperatures for $1 \mathrm{~h}$. It has to be mentioned that the undercooling has not been measured with respect to the thermodynamic melting corresponding to the melting of an infinite-sized crystal since the P3AT films grown by spin-coating are in a kinetically trapped state that is very far from the thermodynamic equilibrium situation. For this reason, we have chosen to refer the undercooling to the experimental melting temperature.

b. Nanomorphology of As-Spin-Coated P3AT Films as Observed by HR-TEM. Representative HR-TEM images recorded in low-dose mode of as-grown thin films of P3HT, P3HeptT, and P3OT are shown in Figure 2. HR-TEM images

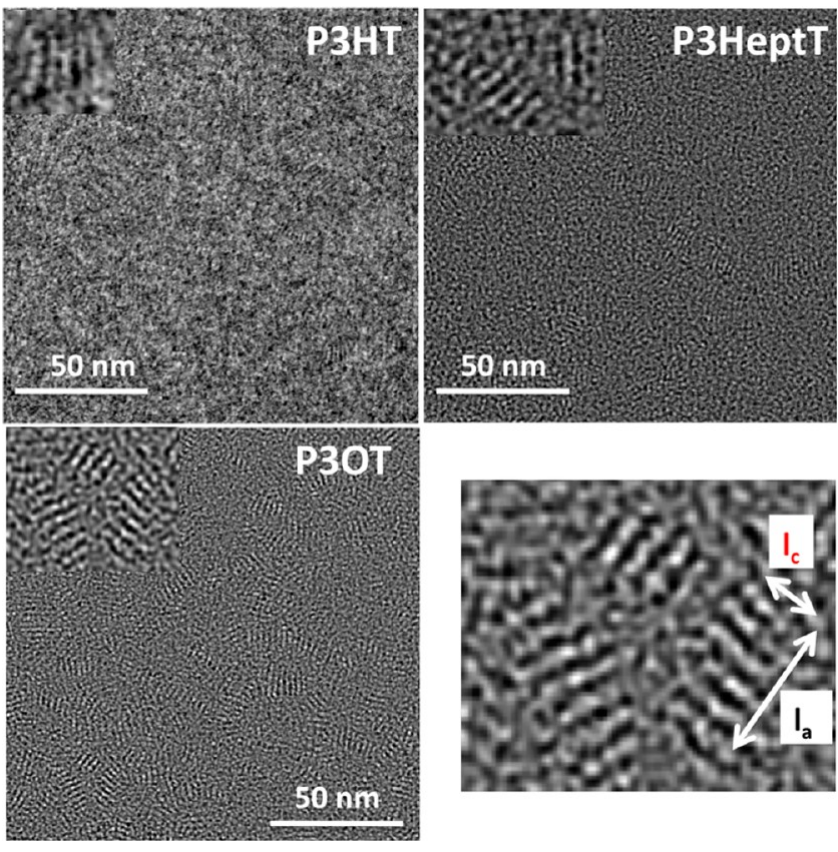

Figure 2. HR-TEM images $\left(150 \times 150 \mathrm{~nm}^{2}\right)$ of as-grown P3AT spincoated films (thickness $\sim 17 \mathrm{~nm}$ ). The insets correspond to enlarged areas of the HR-TEM images and show P3AT nanocrystals. The inset HR-TEM image in the lower right-hand corner illustrates the definition of $l_{\mathrm{a}}$ and $l_{\mathrm{c}}$ (see text) for P3OT nanocrystals.

allow to distinguish between crystalline nanodomains of P3ATs properly oriented with respect to the incident electron beam and the featureless zones that are ascribed to both amorphous interlamellar zones and crystalline domains whose orientation differs from a strict face-on orientation on the substrate. The crystalline domains of these P3ATs are revealed by periodic fringed patterns corresponding to regular $\pi$-stacked chains with a characteristic lamellar period ( $\mathbf{a}_{\mathrm{P} 3 \mathrm{AT}}$ axis). The contrast in the HR-TEM images is related to the presence of sulfur atoms in the stacks of polythiophene backbones versus carbon/hydrogen atoms in the layers of alkyl side chains. These nanocrystals show a preferential face-on orientation on the $\mathrm{Si} / \mathrm{SiO}_{2}$ substrate. The areas not showing any periodic lamellar patterns correspond either to the amorphous zones or to P3AT domains whose orientation to the electron beam prevents their observation by HR-TEM, i.e., as soon as the $\pi$-stacking direction of P3HT chains is no longer normal to the film substrate. This indicates that HRTEM provides only a lower limit of domain size along the $\mathbf{a}_{\mathrm{P} 3 \mathrm{AT}}$ axis as the contrast can be lost for a slight misorientation of the crystalline domain to the substrate normal. More generally, attempts to observe edge-on oriented P3AT crystallites by lowdose HR-TEM were not successful (presumably because of the monoclinic unit cell of P3ATs as evidenced for form I of $\mathrm{P} 3 \mathrm{HT}) .^{34}$ 
The lamellar periods $\lambda_{\mathrm{a}}$ were extracted from the FFTs of the HR-TEM images for the different P3AT samples. As expected, $\lambda_{\mathrm{a}}$ increases linearly with the number of carbon atoms in the side chain, i.e., $1.67 \mathrm{~nm}$ (P3HT), $1.85 \mathrm{~nm}$ (P3HeptT), and $2.0 \mathrm{~nm}$ (P3OT). These periods correspond well with the ones extracted from the angularly averaged ED line scans in Figure 3 as well as the lamellar periods reported in the literature for form I P3ATs (vide infra). ${ }^{5,35}$

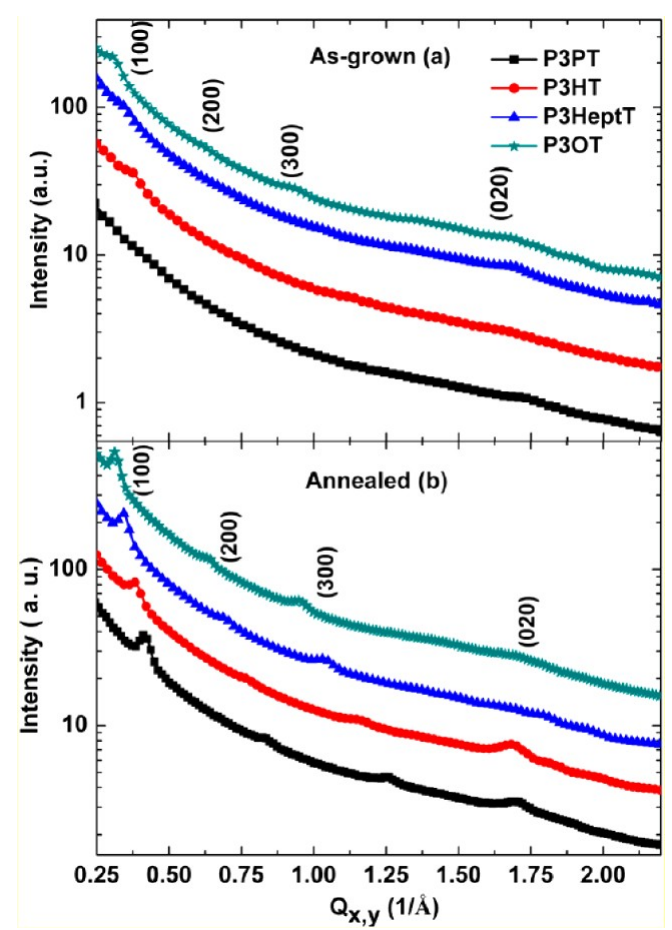

Figure 3. ED line profiles of as-grown (a) and isothermally annealed (b) P3AT films (15-18 nm thick). For clarity, the scans of the different samples were shifted along the ordinate axis.

As seen in Figure 2, the length of the side chains has a clear influence on the proportion of nanocrystals observed by HRTEM. As the length of the alkyl side chains increases, a higher fraction of face-on oriented nanocrystallites is evidenced. This is particularly true for the P3OT films showing well-defined nanocrystallites with face-on orientation. It was possible to perform a statistical analysis of the domain sizes only for the asgrown films showing enough well-defined crystalline domains, i.e., for P3HeptT and P3OT. For each sample, at least five different HR-TEM images were recorded on different locations of the film to construct the statistics of the P3AT nanocrystal dimensions. Two characteristic in-plane dimensions were derived from the HR-TEM images: the average stem length $\left(l_{c}\right)$ and the average domain size perpendicular to the polythiophene backbone, i.e., along the $\mathbf{a}_{\mathrm{P} 3 \mathrm{AT}}$ axis $\left(l_{\mathrm{a}}\right)$. Table 2 collects the corresponding dimension of nanocrystallites.

Globally, the HR-TEM images of as-spin coated P3AT films suggest two conclusions: (i) the lateral size of face-on oriented domains along the $\mathbf{a}_{\mathrm{P} 3 \mathrm{AT}}$ axis tends to increase with increasing number of carbon atoms in the alkyl side chain, and (ii) face-on orientation in the as-prepared films is favored for longer alkyl side chains. However, in the spin-coated films, even for the P3OT films showing the highest proportion and largest face-on oriented nanocrystals, the dimensions of the nanocrystals remain very small, i.e., a few stems $(<5)$ along the $\mathbf{a}_{\mathrm{P} 3 \mathrm{OT}}$ axis direction and less than 20 monomer units along $\mathbf{c}_{\mathrm{P} 3 \mathrm{OT}}$.

The latter observations can be further verified by comparing the angularly averaged ED patterns yielding characteristic line scans for the different P3AT films (see Figure 3). Indeed, whereas for as-grown P3PT only the peak related to the $\pi$ stacking period at $q=1.72 \AA^{-1}$ is observed, P3OT films show both the sequence of $(h 00)$ reflections $(h=1,2$, and 3$)$ as well as a broad reflection corresponding to the characteristic $\pi$-stacking period. This result is further in agreement with the observations of Payerne et al. obtained for P3ATs grown on HOPG graphite: an increasing fraction of face-on oriented crystallites is observed while increasing the alkyl side chain length from hexyl (P3HT $\approx$ $25 \%$ ) to dodecyl (P3DDT $\approx 75 \%) .{ }^{27}$ These observations suggest that both the kinetics of P3AT nanocrystal growth and the interactions with the $\mathrm{SiO}_{2}$ substrate depend on the length of the $n$-alkyl side chains.

c. Impact of Isothermal Annealing: Effect of Alkyl Side Chain Length. All the spin-coated P3AT films were subjected to isothermal annealing using the same undercooling $\Delta T=45^{\circ} \mathrm{C}$ and the same annealing duration $(1 \mathrm{~h})$. Figure 4 depicts the corresponding HR-TEM images of the different P3AT films, whereas Figure $3 \mathrm{~b}$ displays the angularly averaged ED patterns showing characteristic in-plane reflections. Table 2 collects the main information gained from the statistical analysis of the P3AT nanocrystal dimensions.

From the statistical analysis of the HR-TEM images after annealing, major changes of the nanomorphology are evidenced. They concern (i) the fraction of face-on oriented domains and (ii) the size of the face-on oriented domains.

For P3PT a few face-on oriented domains are clearly visible after annealing, but the overall fraction remains low (a few percent). For longer side chains $(n \geq 6)$, there is a clear increase in both size of the domains and fraction of face-on oriented domains. P3OT films show the highest coverage of face-on oriented nanocrystals $(20-30 \%$ of total film surface). Although the determination of the surface density of nanocrystals of P3OT in as spin-coated films is hampered by their very small size, we

Table 2. Average Nanocrystal Dimensions $\left(l_{\mathrm{c}}\right.$ and $\left.l_{\mathrm{a}}\right)$ Obtained from the Statistics on HR-TEM Micrographs on Spin-Coated rrP3AT Thin Films ${ }^{a}$

\begin{tabular}{|c|c|c|c|c|c|c|c|c|}
\hline \multirow[b]{2}{*}{ polymers } & \multicolumn{2}{|c|}{$\begin{array}{l}\text { av crystallite size along the alkyl } \\
\text { side chain (a axis) }\left(l_{\mathrm{a}}\right)(\mathrm{nm})\end{array}$} & \multicolumn{2}{|c|}{$\begin{array}{l}\text { av stem length in the crystallites } \\
\qquad\left(l_{c}\right)(\mathrm{nm})\end{array}$} & \multicolumn{2}{|c|}{ mean area of the crystallites $\left(\mathrm{nm}^{2}\right)$} & \multicolumn{2}{|c|}{$\begin{array}{c}\% \text { of surface covered by face-on } \\
\text { oriented domains }\end{array}$} \\
\hline & as-grown & annealed & as-grown & annealed & as-grown & annealed & as-grown & annealed \\
\hline P3PT & & 11 & & 6.8 & & 98.1 & & 2.6 \\
\hline P3HT & & 12.4 & & 7.8 & & 144.7 & & 5.9 \\
\hline P3HeptT & 7.5 & 15.9 & 5.7 & 8.6 & 57.8 & 207.2 & 0.8 & 15.2 \\
\hline P3OT & 10.4 & 21.6 & 7.1 & 9.4 & 91.1 & 261.2 & 3 & 20.4 \\
\hline
\end{tabular}

${ }^{a}$ For all P3AT's, the annealing temperature corresponds to an undercooling $\Delta T=45^{\circ} \mathrm{C}$ below the experimental melting temperatures. 

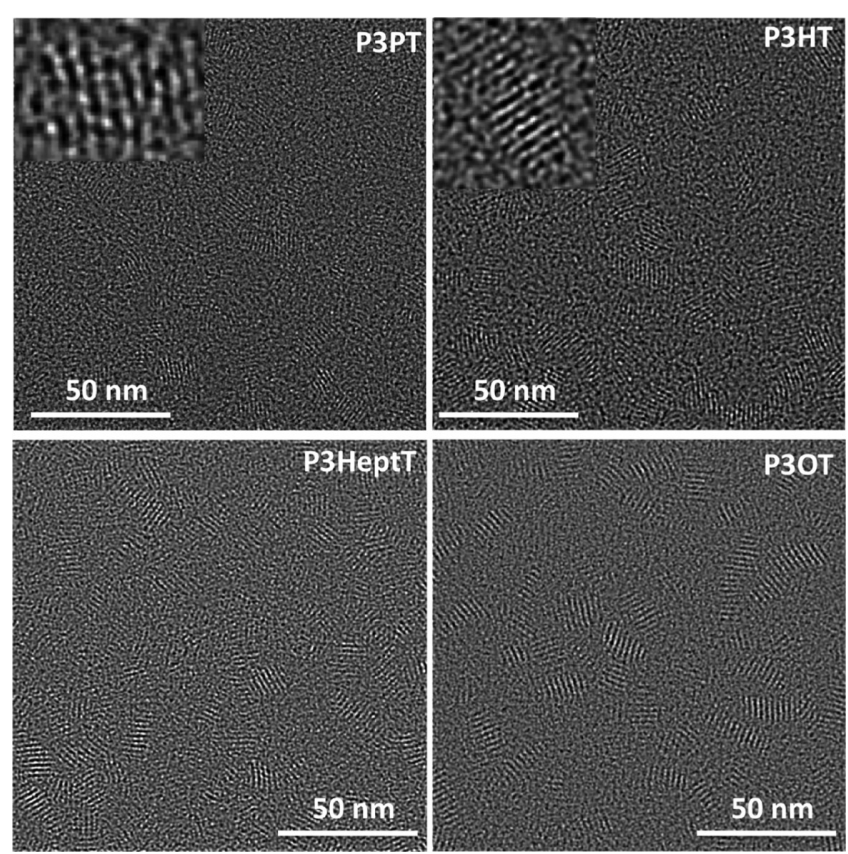

Figure 4. HR-TEM images of spin-coated P3AT films $(\approx 15-18 \mathrm{~nm}$ thick) after isothermal annealing. All films were annealed at a temperature $T_{\mathrm{a}}$ corresponding to an undercooling $\Delta T=45^{\circ} \mathrm{C}$ with respect to the experimental melting temperature.

have estimated it before and after annealing. If the dimensions of the P3OT nanocrystals tend clearly to increase after annealing, the density of nanocrystals per unit surface shows a moderate $30-35 \%$ increase. This indicates that thermal annealing promotes the growth of crystalline P3AT domains out of the amorphous surrounding whereas nucleation of new nanocrystals is limited.

HR-TEM allows for a careful examination of the average domain size after annealing along the chain direction and in the direction of the alkyl side chain. The average $l_{\mathrm{a}}$ and $l_{\mathrm{c}}$ of the crystalline domains were extracted from a set of HR-TEM images, and the mean values are given in Table 2. The statistical analysis of domains sizes after annealing demonstrates a preferential growth of the crystallites along the $\mathbf{a}_{\mathrm{P} 3 \mathrm{AT}}$ axis, i.e., along the alkyl side chain direction. For instance, in the case of P3OT, $l_{\mathrm{a}}=10 \mathrm{~nm}$ before annealing, whereas after annealing $l_{\mathrm{a}}=$ $22 \mathrm{~nm}$. Similarly, for P3HeptT, $l_{\mathrm{a}}$ increases by a factor of almost 2 after annealing. Contrary to what is observed for $l_{a}$, the stem length $l_{c}$ increases only marginally. For P3OT, $l_{c}$ increases from 7 to $9.4 \mathrm{~nm}$ upon annealing. A similar trend is observed for P3Hept T and P3HT. It must be stressed that the width of the P3AT domains observed by HR-TEM in spin-coated films, even after annealing, lies well below that reported for solution-grown P3HT nanofibrils in the range $15-25 \mathrm{~nm}^{36-39}$ Table 2 suggests also that the average stem length in the P3AT nanocrystals after annealing tends to increase with increasing length of the side chains, e.g., $6.8 \mathrm{~nm}$ for P3PT versus $9.4 \mathrm{~nm}$ for P3OT. This difference may be ascribed to a higher "flexibility" of P3OT versus P3PT chains. It also suggests that the side-chain ordering process promotes the ordering of the polythiophene backbone.

The evolution of the nanomorphology by HR-TEM is further substantiated by the evolution of the ED patterns. Figure $3 \mathrm{~b}$ depicts the ED line scans for the different P3AT films after isothermal annealing. For all samples, the intensity of $(h 00)$ and (020) Bragg reflections increases very clearly after the postgrowth annealing. The diffraction profile of annealed P3PT films evidence the presence of both face-on and edge-on oriented crystallites. On the other hand, the annealed P3OT films show strong $(h 00)$ reflections arising from the face-on domains and an ill-defined hump at the position of the $\pi$-stacking period (020) corresponding to edge-on oriented crystallites. More generally, the intensity of the (020) peak tends to decrease as the side chain length increases. This result clearly supports the HR-TEM findings; i.e., the longer the alkyl side chain, the higher the proportion of face-on oriented nanocrystallites.

The HR-TEM images in Figure 4 for P3OT also show that adjacent nanocrystals with a flat-on orientation show high-tilt grain boundaries as evidenced for instance in the case of pentacene nanocrystals. ${ }^{40}$ Such high-tilt grain boundaries are known to be detrimental to the charge transport in thin films of semiconducting materials. ${ }^{40}$ This observation implies also that there is apparently no orientational correlation between the stem directions in adjacent P3OT nanocrystals in the spin-coated films. This is a major difference with respect to the P3HT films oriented by directional epitaxial crystallization (DEC) in 1,3,5trichlorobenzene that show a periodic arrangement of oriented lamellar crystals sharing a common in-plane orientation of the polymer stems. $^{21,22}$

d. Evolution of the Semicrystalline Nanomorphology in P3OT and P3PT Films with Increasing Annealing Temperature. A more detailed analysis of the impact of annealing temperature $T_{\mathrm{a}}$ on the nanomorphology of the spincoated films was performed for P3OT and P3PT.

The nanomorphology of the as-grown and annealed P3OT thin films for different values of $T_{\mathrm{a}}$ are shown in Figure 5 (first column) as well as the corresponding histograms obtained from the statistical analysis of the domain sizes $l_{\mathrm{a}}$ and $l_{\mathrm{c}}$. Whereas few nanocrystals are visible in the as-grown P3OT film (Figure 2a), isothermal annealing at $\Delta T=45^{\circ} \mathrm{C}\left(T_{\text {is }}=139{ }^{\circ} \mathrm{C}\right)$ results in a clear increase of domain size as seen in Figure 5. Comparing the HR-TEM images of as-grown and annealed films, the crystallites grow mainly along the alkyl side-chain direction. The statistical analysis of domain sizes indicates that the length of the crystallites $l_{\mathrm{a}}$ increases by $\approx 3 \mathrm{~nm}$ when $T_{\mathrm{a}}$ increases from 130 to $150{ }^{\circ} \mathrm{C}$ (maximum X-ray intensity for P3OT, Figure 1), whereas $l_{\mathrm{c}}$ increases only marginally. The average stem length extracted from the HR-TEM images for different values of $T_{\mathrm{a}}$ lies in the range $7-9 \mathrm{~nm}$ (Table 2). It is similar to the values reported for P3HT films grown by DEC. ${ }^{21}$ In addition, the HR-TEM images show that the boundaries of the P3OT nanocrystals are sharp but indicate some characteristic tapering as was observed for instance in the case of poly(9,9-di- $n$-octyl-2,7-fluorene) (PFO) and poly(9,9-bis(2-ethylhexyl)fluorene-2,7-diyl) (PF2/ 6) lamellar crystals. ${ }^{41,42}$ In most of the nanocrystals, the domain boundary is not orthogonal to the stem direction. The same observation was made in the case of epitaxied thin films, and it was interpreted in terms of specific crystallographic fold planes. ${ }^{21}$

The same analysis has been carried out for P3PT. Figure 6 depicts the nanomorphology of the P3PT films after annealing for $1 \mathrm{~h}$ at different temperatures, i.e., at $T_{\mathrm{a}}=150{ }^{\circ} \mathrm{C}, 204^{\circ} \mathrm{C}$ and $220^{\circ} \mathrm{C}$, respectively, as well as the corresponding histograms of the domain size in the direction perpendicular to the stems, i.e., $l_{\mathrm{a}}$. Because as-grown films did not provide any quantitative information, the morphology found at $150{ }^{\circ} \mathrm{C}$ was used as a reference. The sizes of the P3PT crystallites are smaller comparatively to P3OT. Despite the higher annealing temperatures for P3PT, the increase in domain size is limited to $\approx 4 \mathrm{~nm}$ along the $\mathbf{a}_{\mathrm{P} 3 \mathrm{AT}}$ axis in the best case $\left(T_{\mathrm{a}}=220{ }^{\circ} \mathrm{C}\right)$. This observation indicates that the increase of domain size is favored 

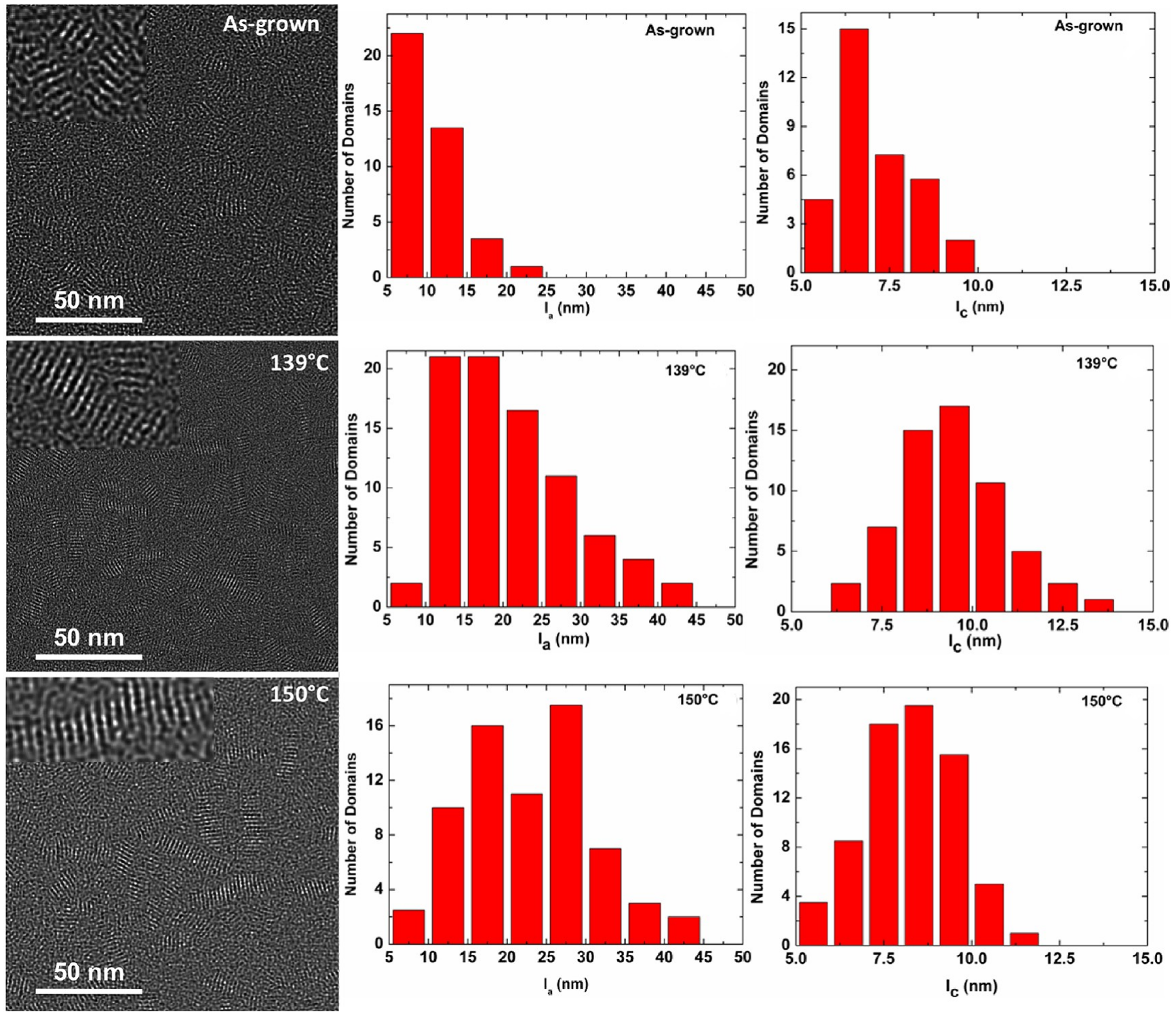

Figure 5. Evolution of the nanomorphology in $\mathrm{P} 3 \mathrm{OT}$ thin films as a function of increasing annealing temperature (middle, isothermal) and corresponding histograms of the domain sizes $l_{\mathrm{a}}$ (lateral dimension along the $\mathbf{a}_{\mathrm{P} 3 \mathrm{OT}}$ axis) and $l_{\mathrm{c}}$ (stem length).

along the side chain direction for longer side chains. However, for both P3PT and P3OT, the increase of domain size along the chain axis direction is limited. In other words, long alkyl side chains favor the growth of the crystalline domains along the side chain direction, i.e., along the $\mathbf{a}_{\mathrm{P} 3 \mathrm{AT}}$ axis, whereas lamellar thickening along the polythiophene chain direction is limited regardless of the length of the alkyl side chains ( $n$-pentyl to $n$ octyl).

Regarding the influence of the annealing temperature on the lateral growth of the crystalline domains, both P3PT and P3OT display the same trend: lateral growth is favored as the annealing temperature increases and approaches a maximum of $l_{\mathrm{a}}$ for an undercooling of $\Delta T=45^{\circ} \mathrm{C}$. This is further supported by GIXD. As seen in Figure $7 \mathrm{a}$, a progressive increase in intensity of the (100) reflection of P3PT with increasing $T_{\mathrm{a}}$ is evidenced.

The same holds for ED line scans shown in Figure $7 \mathrm{~b}$ corresponding to the angularly average ED patterns of P3PT thin films annealed at various temperatures. The ED line scans of asgrown P3PT films are almost featureless, indicating a poor level of crystallinity as evidenced by HR-TEM. Upon annealing, the family of (h00) ( $h=1,2$, and 3$)$ and the (020) reflection related to the $\pi$-stacking emerges. Of importance, a remarkable shift of the (020) peak is observed with increasing $T_{\mathrm{a}}$ for P3PT. The peak shifts from 1.75 to $1.68 \AA^{-1}$ while increasing the annealing temperature from 150 to $204{ }^{\circ} \mathrm{C}$. This shift corresponds to a change of the $\pi$-stacking distance from 3.6 to $3.8 \AA$. No such behavior was observed for the other P3ATs investigated herein. It points at a possible structural modification in P3PT films with increasing annealing temperature, e.g., a specific polymorphism of P3PT. Noteworthy, a similar shift was observed for $40 \mathrm{~nm}$ thick drop-cast films whereas it was absent for thicker layers.

The impact of annealing on the orientation of P3AT crystallites was further investigated by GIXD. This technique is complementary to TEM as it gives important information on the ordering along the out-of-plane and in-plane directions. 2D GIXD patterns of as-spin-coated P3AT films are shown in Figure 8. P3AT thin films were annealed at $\Delta T=45^{\circ} \mathrm{C}$ with respect to the corresponding experimental melting temperatures. The respective 2D GIXD patterns are shown in Figure 9 as well as the line scans along $Q_{x y}$ and $Q_{z}$. 


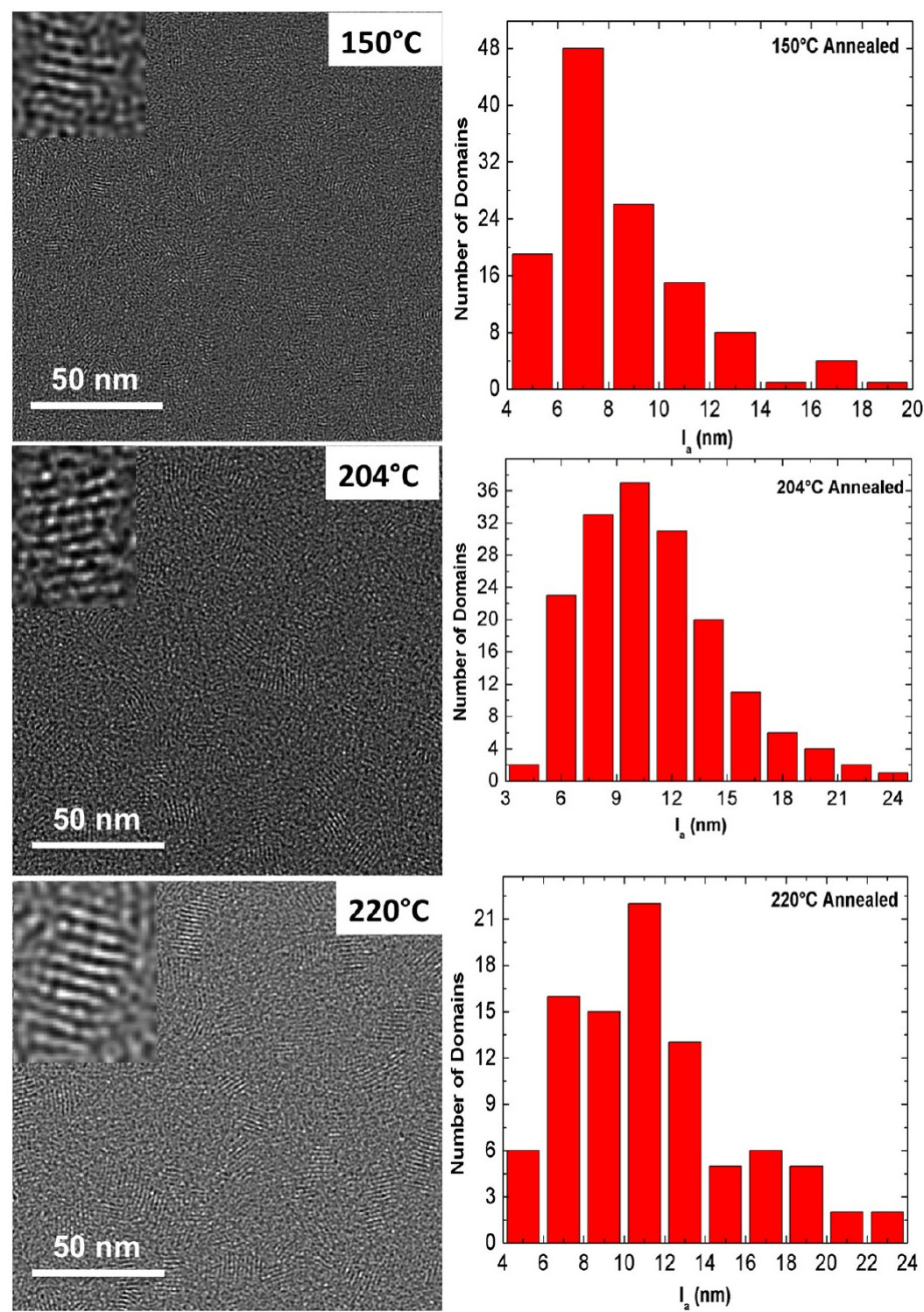

Figure 6. Evolution of the nanomorphology in P3PT thin films (15-18 nm thick) as a function of increasing annealing temperature (middle, isothermal) and corresponding histograms of the domain sizes $l_{\mathrm{a}}$ (lateral dimension along the $\mathbf{a}_{\mathrm{P} 3 \mathrm{PT}}$ axis).

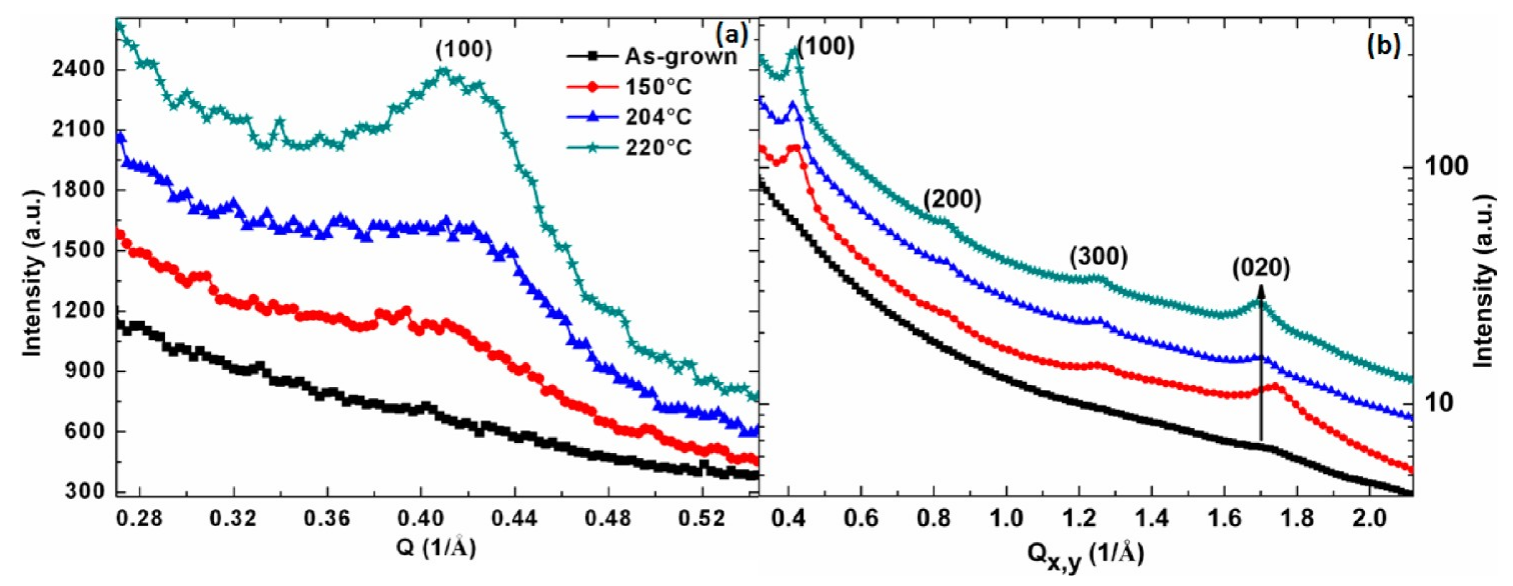

Figure 7. (a) Out-of-plane GIXD line scans of the (100) reflection of P3PT (17 nm thick) for different annealing temperatures. (b) ED integrated line profile of a P3PT film (17 nm thick) annealed at different temperatures. For clarity, the linear scans were shifted along the ordinate axis.

Similar to HR-TEM results we could not observe any Bragg reflections for the as-grown P3PT film, suggesting poor crystallinity (Figure 8a). Regarding P3OT, two Debye-Scherrer rings are observed for as spin-coated films corresponding to 


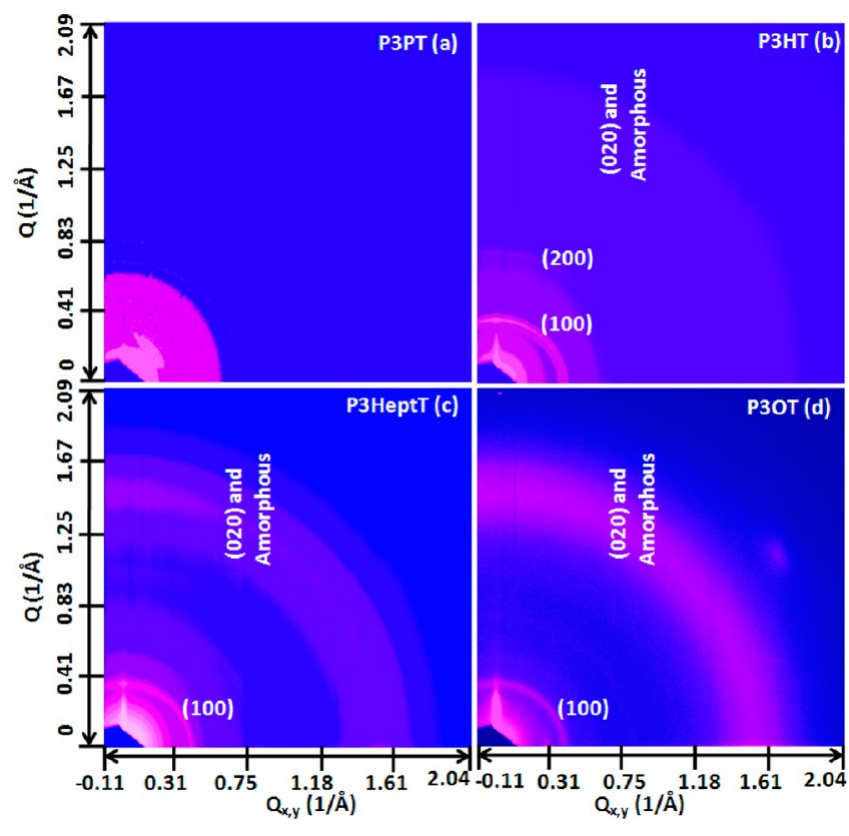

Figure 8. 2D X-ray diffraction patterns of spin-coated P3AT films $(\approx 17$ $\mathrm{nm}$ thick) before annealing.

(100) and (020) reflections. This pattern indicates that P3OT crystallites have no preferred contact plane, in agreement with the ED pattern shown in Figure 3a.

After the postgrowth annealing (Figure 9c,d) all P3ATs show a well-defined (020) reflection along the $Q_{z}$ direction. This indicates that annealing improves substantially the stacking of polythiophene backbones along the $\pi$-stacking direction $\left(b_{\mathrm{P} 3 \mathrm{AT}}\right.$ axis). The nearly isotropic distribution of crystallites becomes textured after the postgrowth annealing. Its angular width is significantly reduced as observed for thicker films. ${ }^{43}$ The (100) reflection is well pronounced along both out-of-plane and inplane directions, confirming the coexistence of edge-on and faceon orientations. Line profiles were extracted from the $2 \mathrm{D}$ patterns along the out-of-plane and in-plane directions as shown in Figures $9 a$ and $9 b$, respectively. The scattering intensity of the (100) reflection along $Q_{x, y}$ is increasing with increasing length of the alkyl side chain (Figure 9b). This observation is consistent with the HR-TEM results showing a higher proportion of face-on oriented P3AT domains and enhanced lateral growth of P3AT domains along $\mathbf{a}_{\mathrm{P} 3 \mathrm{AT}}$ when going from $n$-pentyl to $n$-octyl side chains. In contrast to this, the out-of plane intensity of the (100) reflection decreases with increasing alkyl chain length.

e. Influence of Alkyl Side Chain Length on the Field Effect Mobility. In order to correlate the structural informations gathered by HR-TEM and GIXD with transport poperties, the field effect mobility was measured for P3AT samples both before and after annealing using an undercooling $\Delta T=45^{\circ} \mathrm{C}$. The influence of the alkyl side-chain length on the mobility of annealed P3AT films is shown in Figure 10. The first

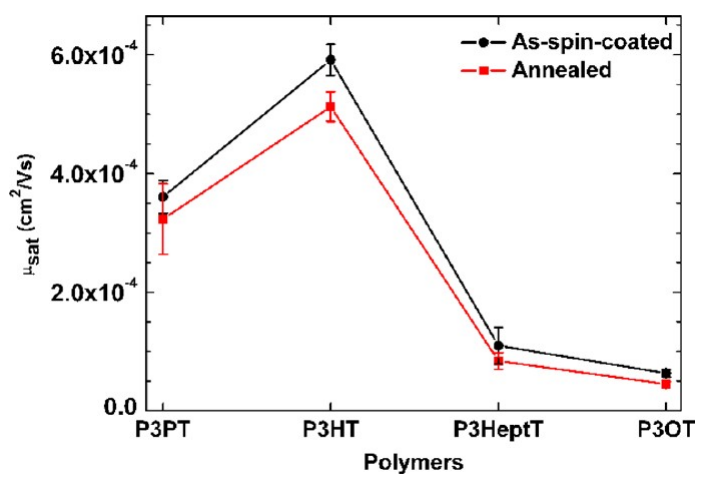

Figure 10. Influence of alkyl side-chain length on the field effect mobility of annealed P3AT OFETs.

remark concerns the impact of annealing. As seen in Figure 10, the charge mobility increases only marginally after annealing, whereas the trend as a function of the side-chain length remains almost identical. Except for P3PT films, the mobility of both annealed and as-deposited P3AT films tends to decrease with
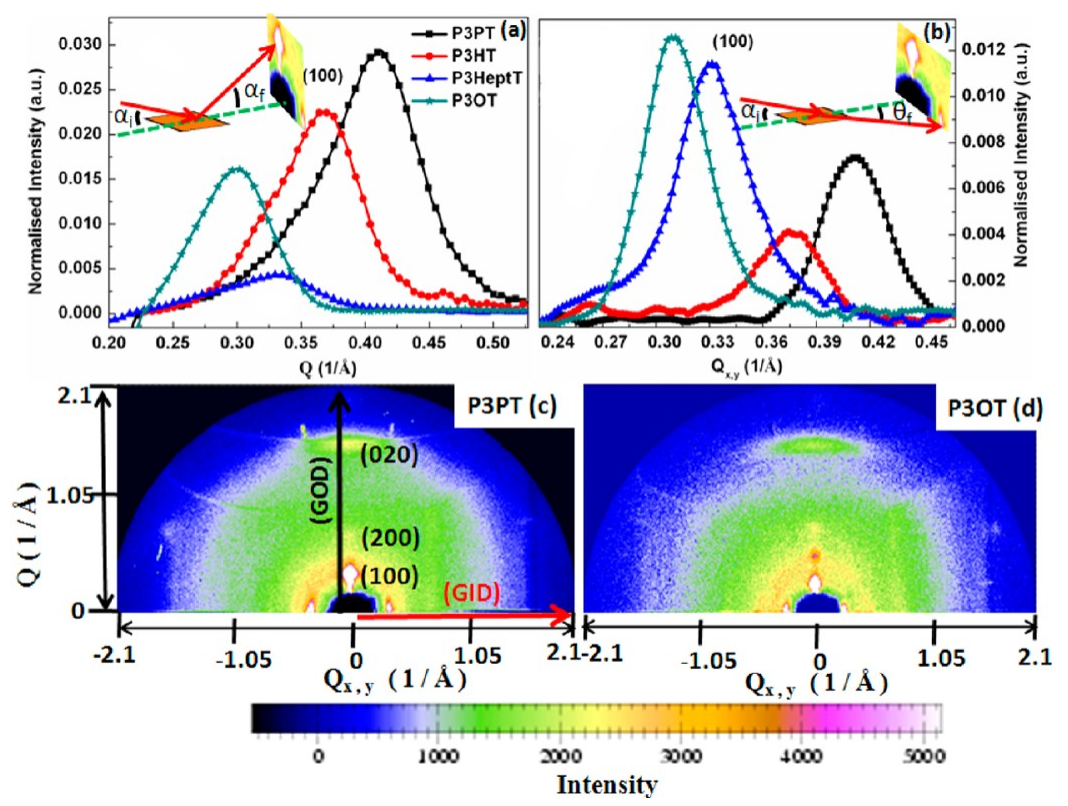

Figure 9. 2D X-ray diffraction maps of P3PT and P3OT spin-coated films $\left(15-20 \mathrm{~nm}\right.$ thick) annealed for $1 \mathrm{~h}$ at $\Delta T=45^{\circ} \mathrm{C}$ and corresponding intensity lines along $Q_{x y}$ and $Q_{z}(\mathrm{a}$ and $\mathrm{b})$. 
increasing length of the alkyl side chain from $6 \times 10^{-4} \mathrm{~cm}^{2} /(\mathrm{V} \mathrm{s})$ for P3HT to $5 \times 10^{-5} \mathrm{~cm}^{2} /(\mathrm{V} \mathrm{s})$ for P3OT. In other words, the mobility of annealed P3HT films is 1 order of magnitude higher comparatively to $\mathrm{P} 3 \mathrm{OT}$ films. This trend is similar to that reported by Park et al. ${ }^{44}$ and Babel et al. ${ }^{45}$ It suggests that the mobility of P3AT films annealed in the same conditions (same $\Delta T$ ) tends to decrease with increasing side-chain length. Moreover, the GIXD and TEM data collected for the same samples show that the proportion of face-on oriented crystallites becomes larger as the alkyl side chain increases from $n$-pentyl to $n$-octyl. Accordingly, the observed decrease of charge mobility may also reflect the decrease of the proportion of edge-on oriented domains in the films when the alkyl side chain increases. $^{1,12}$

\section{DISCUSSION}

Various groups have evidenced the importance of macromolecular parameters (regioregularity and molecular weight) of P3AT's on the formation of edge-on and face-on oriented crystallites and the correlations with the performances of solar cells and OFETs. ${ }^{17,8,18,30}$ Regarding morphology of spin-coated P3HT films, nanofibrils are most often observed via $\mathrm{AFM}^{4,16,17}$ Annealing is reported to favor the growth of long P3HT nanofibrils. ${ }^{15,17}$ Cho et al. reported a 2 -fold increase of charge mobility upon annealing a P3HT $\left(M_{w}=40 \mathrm{kDa}\right)$ dip-coated film at $150{ }^{\circ} \mathrm{C}$ for $15 \mathrm{~min} .{ }^{19} \mathrm{Zen}$ et al. found a similar increase of mobility for the chloroform fraction of P3HT after annealing at $150{ }^{\circ} \mathrm{C} .{ }^{14}$

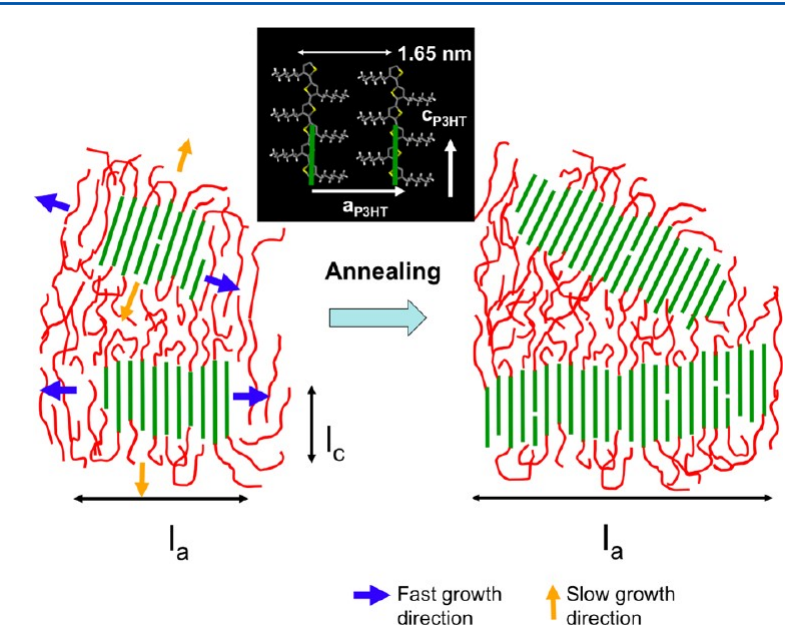

Figure 11. Schematic showing the anisotropy of P3AT lamellar growth. Annealing promotes fast growth along the side chain direction whereas lamellar thickening remains limited.

However, AFM does not allow to map the structure of the domains down to the individual stacks of polymer chains. Nanofibrils observed by AFM may be polycrystalline to a certain extent. In this context, HR-TEM provides a complementary view of the nanomorphology in spin-coated and annealed layers for various P3ATs. In the following, we will discuss the main findings using the frame of polymer crystallization established for more classical systems, e.g. polyolefins.

In most semicrystalline polymers, e.g. PE, annealing can result in lamellar thickening (increase of the stem length in the lamellae) or lateral growth (increase of the number of stems in the direction perpendicular to the chains) as illustrated in Figure 10. Lamellar thickening occurs via reorganization of polymer chains within stacked lamellae, i.e., a process of secondary crystallization. ${ }^{46,47}$ In other words, during thickening, no new crystalline chains are introduced in the crystal from the surrounding melt. As opposed to this, lateral growth implies the incorporation of new chains in a crystal. The experimental data gathered in this study show that both lamellar thickening and lateral growth depend on the length of the alkyl side chains.

More generally, annealing of P3AT films does not induce a substantial thickening of the crystalline domains along the polythiophene backbone direction, at least within the frame of annealing duration used in this study. Moreover, increasing the annealing temperature $T_{\mathrm{a}}$ does not further improve lamellar thickening substantially. As a matter of fact, the maximum stem length measured in annealed films of P3OT is comparable to that observed for P3HT films grown by directional epitaxial crystallization (DEC), i.e. $9 \mathrm{~nm} .{ }^{21}$ Only the P3HT films grown very slowly by DEC at $20 \mu \mathrm{m} / \mathrm{s}$ show a higher average stem length of $13 \mathrm{~nm}$ as measured by Hartmann et al. ${ }^{22}$ This result suggests that the slide-diffusion of the polythiophene backbones within the existing nanocrystals is particularly difficult during the thermal annealing which results in limited lamellar thickening. This is not unexpected as the $\pi-\pi$ interactions between the P3AT stems are much stronger than the van der Waals interactions between PE chains in a PE crystal. Accordingly, the energetic barrier to the sliding of a polythiophene chain in a P3AT nanocrystal is most presumably much larger as compared to PE, and hence lamellar thickening is limited.

However, the present results indicate that annealing favors the growth of P3AT nanocrystals along both the side-chain direction, i.e. $\mathbf{a}_{\mathrm{P} 3 \mathrm{AT}}$, and along the $\pi$-stacking direction, $\mathbf{b}_{\mathrm{P} 3 \mathrm{AT}}$. This "lateral" growth is all the more pronounced as the side chains are longer. Lateral growth is most efficient for P3OT, i.e., $n$-octyl side chains as opposed to P3PT with shorter $n$-pentyl side chains. In the range of investigated annealing temperatures, the layers of alkyl side chains are in a disordered state, and the ordering process is that of a smectic-like systems made of planes of strongly interacting $\pi$-stacked polythiophene chains. The origin of the efficient lateral growth of the P3AT lamellae with increasing number of carbon atoms in the side chain can be twofold: (i) it might be due to an easier folding ability of the P3AT chains and (ii) a higher overall plasticity of the crystalline lattice.

As demonstrated by several authors, P3HT crystallized from solution shows an onset of folding for $M_{\mathrm{w}} \approx 10 \mathrm{kDa} .^{28,29}$ For all investigated P3ATs in this study, the $M_{\mathrm{w}}$ of the sample implies the possibility for folding of the polythiophene backbone. As indicated in earlier studies, in order to form a "tight" fold, the P3AT chain must show a sequence of about 8 thiophene units in cis conformation. The tightest fold implies a folding diameter of $\sim 2.0 \mathrm{~nm}$. In P3PT, two chains are separated by $1.51 \mathrm{~nm}$ along the $\mathbf{a}_{\mathrm{P} 3 \mathrm{PT}}$ axis versus $2.0 \mathrm{~nm}$ for P3OT. This suggests that tight folds form more readily in $\mathrm{P} 3 \mathrm{OT}$ as compared to $\mathrm{P} 3 \mathrm{PT}$, enhancing the lateral growth along $\mathbf{a}_{\mathrm{P} 3 \mathrm{AT}}$ upon annealing of P3OT nanocrystals.

A second reason explaining the improved lateral growth for higher number of carbon atoms in the side chains is certainly related to the overall plasticity of the crystal conveyed by the side chains. For the annealing temperatures used in this study, the lateral side chains are in a disordered "molten" state whereas strong $\pi-\pi$ interactions maintain the structural cohesion of the lamellae made of $\pi$-stacked polythiophene backbones. ${ }^{13}$ Lateral growth of P3AT lamellae occurs via addition of chains at the growth front of the lamellae, i.e., at the two terminal $(b, c)$ planes of the lamellae. Mobility of chains in the vicinity of the growth front is expected to be higher for P3AT's bearing long side chains. 
Along the same line, Causin et al. reported that P3DDT is crystallizing faster than poly(3-butylthiophene) because of the increased molecular mobility in the melt as the side-chain length increases. $^{48}$ This explains also the observation of a lower overall crystallinity in as spin-coated layers of P3ATs for shorter side chains. This limited molecular mobility may also account for the lower crystallinity in as spin-coated P3AT films with the shortest side chains.

From the point of view of charge transport, mobility is determined by both the intrinsic transport within crystalline domains and the interdomain transport which is limited by the interconnectivity through the amorphous via tie chains, chain folds, and tie crystallites. Regarding charge transport within crystalline domains, it is the extension of the domains along the chain direction ( $c_{\mathrm{P} 3 \mathrm{AT}}$ axis) that is expected to lead to improved charge transport. However, the present results show that annealing promotes mainly the growth of the nanocrystals along the $\mathbf{a}_{\mathrm{P} 3 \mathrm{AT}}$ axis (direction of the side chains), i.e., along the direction of poor charge transport, whereas the stem length in the crystalline domains increases only very little. Accordingly, transport of single nanocrystals is not expected to be substantially improved upon annealing. In addition, the higher fraction of faceon oriented domains after annealing is also unfavorable for inplane charge transport. Regarding transport between P3AT nanocrystals, annealing is shown to improve only marginally the total crystallinity as can be inferred from the increase of the fraction of face-on domains in the HR-TEM images (at best $20 \%$ of the total surface for P3OT). Accordingly, the structural results obtained by HR-TEM and GIXD are consistent with the very limited increase of field effect mobility observed after annealing.

\section{CONCLUSION}

HRTEM and GIXD have been used to follow the nanomorphology of semicrystalline P3AT thin films upon annealing. Statistical analysis of HR-TEM images allows to quantify the characteristic dimensions of the P3AT nanocrystals (average stem length $l_{\mathrm{c}}$ and lateral dimension perpendicular to the stems $l_{\mathrm{a}}$ ). Clear trends are evidenced when the number of carbon atoms in the alkyl side chain increases: (i) the proportion of face-on oriented nanocrystals increases; (ii) the lateral growth of P3AT nanodomains along the side chain direction is enhanced. The longer side chains confer a higher overall plasticity of P3AT chains and favor the growth of face-on oriented P3AT nanocrystals on $\mathrm{SiO}_{2}$ substrate during annealing. Lateral growth along the side-chain direction $\mathbf{a}_{\mathrm{P} 3 \mathrm{AT}}$ is favored for higher annealing temperatures contrary to lamellar thickening that remains marginal. Lamellar thickening is hampered by the limited slide-diffusion of P3AT chains within layers of $\pi$-stacked polythiophene backbones. Lateral growth is eased by the weak van der Waals interactions between layers of $\pi$-stacked polythiophene backbones along the alkyl side-chain direction $\mathbf{a}_{\text {Р3AT }}$. Despite a sizable growth of P3AT nanocrystals along $\mathbf{a}_{\mathrm{P} 3 \mathrm{AT}}$ after annealing, the absence of substantial lamellar thickening results in a poor increase of the field effect mobility in the films.

\section{AUTHOR INFORMATION}

\section{Corresponding Author}

*E-mail: martin.brinkmann@ics-cnrs.unistra.fr.

\section{Notes}

The authors declare no competing financial interest.

\section{ACKNOWLEDGMENTS}

Bernard Lotz is gratefully acknowledged for enlightening discussions and comments. The authors are thankful to Ms. Anna Bergen for UV-vis absorption spectroscopic analysis and beamline scientist from BL9, DELTA, and P08, PETRA III for their support during the experiment. T.S.S. is thankful to DAAD and S.G. and U.P. to BMBF for financial support.

\section{REFERENCES}

(1) So, F. Organic Electronics: Materials, Processing, Devices and Applications; CRC: Boca Raton, FL, 2009.

(2) Wong, W. S.; Salleo, A. Flexible Electronics: Materials and Applications; Springer-Verlag: Berlin, 2009.

(3) Prosa, T. J.; Winokur, M. J.; Moulton, J.; Smith, P.; Heeger, A. J. Macromolecules 1992, 25, 4364-4372.

(4) Prosa, T. J.; Winokur, M. J.; McCullough, R. D. Macromolecules 1996, 29, 3654-3656.

(5) Brinkmann, M. J. Polym. Sci., Part B: Polym. Phys. 2011, 49, 1218.

(6) Shabi, T. S.; Grigorian, S.; Brinkmann, M.; Pietsch, U.; Koenen, N.; Kayunkid, N.; Scherf, U. J. Appl. Polym. Sci. 2012, 2335.

(7) Yang, H.; Lefevre, S. W.; Ryu, C. Y.; Bao, Z. Appl. Phys. Lett. 2007, 90, 172116

(8) Salleo, A.; Kline, R. J.; DeLongchamp, D. M.; Chabinyc, M. L. Adv. Mater. 2010, 22, 3812.

(9) Brinkmann, M.; Wittmann, J. C. Adv. Mater. 2006, 18, 860.

(10) Sirringhaus, H.; Brown, P. J.; Friend, R. H.; Nielsen, M. M.; Bechgaard, K.; Langeveld-Voss, B. M. W.; Spiering, A. J. H.; Janssen, R. A. J.; Meijer, E. W.; Herwig, P.; de Leeuw, D. M. Nature 1999, 401, 685688.

(11) Lu, G.; Li, L.; Yang, X. Adv. Mater. 2007, 19, 3594.

(12) Kline, R. J.; McGehee, M. D.; Toney, M. F. Nat. Mater. 2006, 5, 222-228.

(13) Joshi, S.; Pingel, P.; Grigorian, S.; Panzner, T.; Pietsch, U.; Neher, D.; Forster, M.; Scherf, U. Macromolecules 2009, 42, 4651.

(14) Zen, A.; Pflaum, J.; Hirschmann, S.; Zhuang, W.; Jaiser, F.; Asawapirom, U.; Rabe, J. P.; Scherf, U.; Neher, D. Adv. Funct. Mater. 2004, 14, 757-764.

(15) Sauvé, G.; Javier, A. E.; Zhang, R.; Liu, J.; Sydlik, S. A.; Kowalewski, T.; McCullough, R. D. J. Mater. Chem. 2010, 20, 3195.

(16) Porzio, W.; Scavia, G.; Barba, L.; Arrighetti, G.; Milita, S. Eur. Polym. J. 2011, 47, 273.

(17) (a) Kline, R. J.; McGehee, M. D.; Toney, M. F. Nat. Mater. 2006, 5, 222-228. (b) Kline, R. J.; McGehee, M. D.; Kadnikova, E. N.; Liu, J.; Frechet, J. M. J.; Toney, M. F. Macromolecules 2005, 38, 3312.

(18) Chang, J. F.; Sun, B.; Breiby, D. W.; Nielsen, M. M.; Sölling, T. I.; Giles, M.; McCulloch, I.; Sirringhaus, H. Chem. Mater. 2004, 16, 4772.

(19) Cho, S.; Lee, K.; Yuen, J.; Wang, G.; Moses, D.; Heeger, A. J.; Surin, M.; Lazzaroni, R. J. Appl. Phys. 2006, 100, 114503.

(20) Wunderlich, B. In Structure, Morphology, Defects in Macromolecular Physics; Academic Press: London, 1973; Vol. 1.

(21) Brinkmann, M.; Rannou, P. Macromolecules 2009, 42, 1125.

(22) Hartmann, L.; Uttiya, S.; Crossland, E.; Kayunkid, N.; Ludwigs, S.; Brinkmann, M. Adv. Funct. Mater. 2011, $21,4047$.

(23) McNeill, C. J. Polym. Sci., Part B: Polym. Phys. 2011, 49, 909.

(24) Mena Osteritz, E. A.; Meyer, B.; Langeveld, M. W.; Voss, R. A.; Janssen, J.; Meijer, E.; Bäuerle, P. Angew. Chem. 2000, 112, 2791.

(25) Deleted in proof.

(26) Grévin, B.; Rannou, P.; Payerne, R.; Pron, A.; Travers, J. P. J. Chem. Phys. 2003, 118, 7097.

(27) Payerne, R.; Brun, M.; Rannou, P.; Baptist, R.; Grevin, B. Synth. Met. 2004, 146, 311.

(28) Liu, J.; Arif, M.; Zou, J.; Khondaker, S. I.; Zhai, L. Macromolecules 2009, 42, 9390.

(29) Brinkmann, M.; Rannou, P. Adv. Funct. Mater. 2007, 17, 101.

(30) Zhai, L.; Pilston, R. L.; Zaiger, K. L.; Stokes, K. K.; McCullough, R. D. Macromolecules 2003, 36, 61.

(31) Loewe, R. S.; Khersonsky, S. M.; McCullough, R. D. Adv. Mater. 1999, 11, 250. 
(32) Loewe, R. S.; Ewbank, P. C.; Liu, J.; Zhai, L.; McCullough, R. D. Macromolecules 2001, 34, 4324.

(33) Braga, D.; Horowitz, G. Adv. Mater. 2009, 21, 1473-1486.

(34) Kayunkid, N.; Uttiya, S.; Brinkmann, M. Macromolecules 2010, 43, 4961.

(35) Meille, S. V.; Romita, V.; Caronna, T.; Lovinger, A. J.; Castellani, M.; Belobrzeckaja, L. Macromolecules 1997, 30, 7898.

(36) Brinkmann, M. J. Polym. Sci., Part B: Polym. Phys. 2011, 49, 1218.

(37) Brinkmann, M.; Chandezon, F.; Julien-Raban, C.; Pansu, R. P. Adv. Funct. Mater. 2009, 19, 2759.

(38) Samitsu, S.; Shimomura, T.; Heike, S.; Hashizume, T.; Ito, K. Macromolecules 2008, 41, 8000 .

(39) Berson, S.; De Bettignies, R.; Bailly, S.; Guillerez, S. A. Adv. Funct. Mater. 2007, 17, 1377.

(40) Drummy, L. F.; Kübel, C.; Lee, D.; White, A.; Martin, D. C. Adv. Mater. 2002, 14, 54.

(41) Brinkmann, M.; Charoenthai, N.; Traiphol, R.; Piyakulawat, P.; Wlosnewski, J.; Asawapirom, U. Macromolecules 2009, 42, 8298.

(42) Brinkmann, M. Macromolecules 2007, 40, 7532.

(43) Joshi, S.; Grigorian, S.; Pietsch, U.; Pingel, P.; Zen, A.; Neher, D.; Scherf, U. Macromolecules 2008, 41, 6800.

(44) Park, Y. D.; Kim, D. H.; Jang, Y.; Cho, J. H.; Hwang, M.; Lee, S. H.; Lim, J. A.; Cho, K. Org. Electron. 2006, 7, 514.

(45) Babel, A.; Jenekhe, S. A. Synth. Met. 2005, 148, 169.

(46) Hikosaka, M.; Amano, K.; Rastogi, S.; Keller, A. Macromolecules 1997, 30, 2067.

(47) Hikosaka, M.; Amano, K.; Rastogi, S.; Keller, A. J. Mater. Sci. 2000, $35,5157$.

(48) Causin, V.; Marega, C.; Marigo, A.; Valentini, L.; Kenny, J. M. Macromolecules 2005, 38, 409. 